EESTI NSV TEADUSTE AKADEEMIA TOIMETISED. XV KÖIDE

FUUSIKA-MATEMAATIKA- JA TEHNIKATEADUSTE SEERIA, 1966, NR. 1

ИЗВЕСТИЯ АКАДЕМИИ НАУК ЭСТОНСКОН ССР. ТОМ ХV СЕРИЯ ФИЗИКО-МАТЕМАТИЧЕСКИХ И ТЕХНИЧЕСКИХ НАУҚ. 1966, № 1

\title{
ИМПУЛЬСНЫИ МЕТОД ИССЛЕДОВАНИЯ СПЕКТРОВ ЯДЕРНОГО МАГНИТНОГО ДВОЙНОГО РЕЗОНАНСА (ЯМДР) ВЫСОКОГО РАЗРЕШЕНИЯ
}

Спектры ядерного магнитного резонанса высокого разрешения обычно снимают при помощи мостовых схем $\left[{ }^{1,3}{ }^{3}\right]$ или скрещенных катушек [2]. Иногда применяют и простой контур, подключенный одновременно к передатчику и приемнику $\left[{ }^{4}, 5\right]$. Отношение сигнал/шум в последнем случае несколько хуже, но зато метод Роллина $\left[{ }^{4}\right]$ отличается простотой и стабильностью и может быть применен в широком диапазоне температур. Кроме того, измерительная головка в этом случае не является микрофонной и мало чувствительна к изменениям электрических характеристик образца. Благодаря этому данный метод часто применяется в промышленных спектрометрах $\left[{ }^{5}\right]$ и датчиках. К сожалению, он не применим тогда, когда на контуре образуется значительное высокочастотное напряжение. В опытах по двойному резонансу $\left[{ }^{6},{ }^{7}\right]$ и в спектрометрии углерода- $13\left[{ }^{8}, 9\right]$ приходится работать с мостами или скрещенными катушками, встречаясь со всеми вытекающими отсюда трудностями.

Метод разделения времени близок к методу Роллина, но отличие состоит в том, что передатчик и приемник попеременно подключают к контуру с образцом, вследствие чего высокочастотная энергия может передаваться от передатчика к приемнику только за счет свободнсй прецессии ядерных спинов. Впервые такая методика была применена Арнольдом в 1957 г. $\left[{ }^{10}\right]$ для исследования спектров высокого разрешения, а также для определения гиромагнитных отношений и времен релаксации [11]. Разделение времени дало хорошие результаты при исследовании спектров монорезонанса в некоторых лабораториях США $[12,14]$ и Қанады $\left[{ }^{13}\right]$, в последнем случае при исследовании спектров фтора. В настоящей работе были исследованы возможности прнменения метода разделения времени для снятия спектров двойного протон-протонного резонанса.

Теория. В отличие от импульсного метода спинового эха, в методс разделения времени применяются длинные высокочастотные импульсы относительно малой интенсивности и исследуется только квазистационарное состояние системы. Наиболее важными характеристиками рассматриваемого метода являются частота повторения $\omega_{m}$ и длина $\tau_{\text {пер }}^{\text {отк }}$ высокочастотных импульсов от передатчика. Оптимальная величина $\omega_{\prime \prime}$ зависит от поставленной задачи, от времен релаксации спиновой системы и от некоторых электрических параметров прибора. Рассмотрим более подробно требования к $\omega_{m}$ в зависимости от времен релаксации.

Будем исходить из обычного гамильтониана для ядерной спиновой системы молекулы $\left[{ }^{15}, 17,21\right]$. Пусть на спиновую систему действует, кроме сильного постоянного магнитного поля $H_{0}$ вдоль оси $z$, сильное возмущающее высокочастотное поле $H_{2}$ и слабое измерительное поле $H_{1}$, оба направленные по оси $x$. Тогда, следуя обозначениям Блоха $\left[{ }^{15}\right]$, имеем 


$$
\begin{gathered}
\hat{n}^{-1} \mathrm{H}(t)=-\sum_{r} \omega_{r} I_{z r}+\sum_{r \neq s} J_{r s} I_{z r} I_{z s}+ \\
+\mathrm{D}_{2}^{+} e^{i \omega_{2} t}+\mathrm{D}_{2}^{-} e^{-i \omega_{2} t}+\mathrm{D}_{1}^{+} e^{i \omega_{1} t}+\mathrm{D}_{1}^{-} e^{-i \omega_{1} t} \\
\mathrm{D} \frac{ \pm}{1,2}=-\frac{1}{2} \gamma H_{1,2} \sum_{r} I_{ \pm} \quad \text { и } H_{2} \gg H_{1} .
\end{gathered}
$$

С помощью преобразования (см. $\left.{ }^{15}\right]$ формула 7.8 и $\left[{ }^{16}\right]$ )

$$
\mathrm{T}=e^{-i \omega_{2} t \sum_{r} I_{z r}}
$$

осуществим переход к вращающимся координатам, в которых отсутствует сильная зависимость гамильтониана от времени.

Если в лабораторных координатах действительно уравнение Шредингера

$$
i \dot{\hbar} \psi=H(l) \psi
$$

то во вращающихся координатах

$$
\begin{aligned}
& i \dot{\hbar} \dot{\psi}_{\mathrm{T}}=\mathrm{H}_{\mathrm{T}} \psi_{\mathrm{T}} \text {, где } \psi_{\mathrm{T}}=\mathrm{T} \psi \text {, } \\
& i \dot{\bar{n}} \dot{\psi}_{\mathrm{T}}=i \dot{\overline{\mathrm{T}}} \dot{\psi}+i \dot{\overline{\mathrm{T}}} \psi=\left(\mathrm{TH}(t) \mathrm{T}^{-1}\right) \mathrm{T} \psi+\left(i \dot{\bar{n}} \mathrm{~T}^{-1}\right) \mathrm{T} \psi=\mathrm{H}_{\mathrm{T}} \psi_{\mathrm{T}} \text {. }
\end{aligned}
$$

Используя зависимости ([15] формула 7.19)

$$
\mathrm{T}^{i} \mathrm{~T}^{-1}=e^{\mp i \omega_{2} t} I_{ \pm}, \mathrm{T} I_{z} \mathrm{~T}^{-1}=I_{z}
$$

и предполагая, что высокочастотное поле $H_{2}$ влияет лишь на переходы ядра $s$ с резонансной частотой $\omega_{s}$, имеем далее

$$
\begin{gathered}
\hbar^{-1} \mathrm{H}_{\mathrm{T}}=-\sum_{r}\left(\omega_{r}-\omega_{2}\right) I_{z r}+\sum_{r \neq s} J_{r s} I_{z r} I_{z s}+\left(\mathrm{D}_{2}^{+}+\mathrm{D}_{2}^{-}\right)+ \\
-\mathrm{D}_{1}^{+} e^{i\left(\omega_{1}-\omega_{2}\right) t}+\mathrm{D}_{1}^{-} e^{-i\left(\omega_{1}-\omega_{2}\right) t} .
\end{gathered}
$$

Зависящие от времени члены уравнения (7) малы и являются лишь слабым возмущением, вызываюшим переходы между стационарными состояниями $\mathrm{H}_{\mathrm{T}}$, однако член

$$
\left(\mathrm{D}_{2}^{+}+\mathrm{D}_{2}^{-}\right)=-\gamma H_{2} I_{x s}
$$

достаточно велик и является причиной появления различных эффектов, свойственных двойному резонансу (расщепления линий, коллапс) $\left[{ }^{17}\right]$. Величина этого недиагонального элемента зависит от силы возмущающего поля. Компоненты вектора $I_{s}$ при этом равны

$$
\left(\omega_{s}-\omega_{2}-J m\right) I_{z s} \text { и } \gamma H_{2} I_{x s} .
$$

Если

$$
\left(\omega_{s}-\omega_{2}-J m\right)>\gamma H_{2}>1 / T_{2 r}^{*}
$$

то наблюдается расщепление спектральных линий, соответствующих ғекоторым переходам ядер $r$, имеющим общие уровни энергии с переходами возмущаемого ядра $s$ (тиклинг). В обратном случае, когда

$$
\gamma H_{2}>\left(\omega_{s}-\omega_{2}-J m\right)
$$

наблюдается подавление спин-спинового взаимодействия (коллапс) $\left[{ }^{17}\right]$. 
Дальнейшие расчеты показывают, что вид спектра двойного резонанса ядер $r$ определяется в основном поперечной компонентой намагниченности возмущаемого ядра $s$. Так как в опытах по методу разделения времени амплитуды $\mathrm{D}_{2}^{+}$и $\mathrm{D}_{2}^{-}$уже не являются постоянными во времени, надо полагать, что мы имеем право использовать приведенные выше результаты только тогда, когда $\mathrm{D}_{2}^{+}$и $\mathrm{D}_{2}^{-}$изменяются настолько быстро, что спиновая система не успевает больше следить за этими изменениями и, таким образом, если

$$
\text { (1) } m \gg 1 / T_{2 s}^{*},
$$

то спектры двойного разонанса имеют обычный вид.

Требования к экспериментальным условиям. Для того, чтобы высоксчастотная энергия могла передаваться от передатчика к приемнику только за счет процессов в ядерной спиновой системе образца, необходимо выполнение условия

$$
\tau=\frac{Q}{2 \pi i} \ll \frac{2 \pi}{\omega_{m}} .
$$

Здесь $Q$ - фактор добротности контура с образцом, $f$ - резонансная частота и $\tau$ - постоянная времени контура. Постоянная времени $\tau$ показывает, за какое время среднее значение напряжения на контуре после выключения передатчика падает в $e=2,718$ раза.

Импульсная работа передатчика соответствует амплитудной модуляции высокочастотного сигнала частотой повторения импульсов $\omega_{m}$ и сопровождается появлением боковых полос на расстоянии $\omega_{m}$ от несущей частоты. Чтобы не вызывать усложнения спектра, эти боковые полосы должны попасть либо за пределы спектра, либо занимать диапазон частот меньший, чем ширина исследуемых линий. Соответственно возможны два случая

$$
\tau \ll 2 \pi / \omega_{m} \ll T_{2 r}^{*}, \quad 1 / \delta,
$$

где $\delta$ - ширина спектра

$$
\tau \ll 2 \pi / \omega_{m} \gg T_{2 r}^{*} .
$$

Первый случай соответствует условиям, когда боковые полосы попадают за пределы спектра. Используя метод разделения времени с диапазоном частоты повторения импульсов $\omega_{m}$, соответствующим условию (14), можно снимать хорошие спектры монорезонанса.

В случае двойного резонанса необходимо выполнение дополнительного требования

$$
2 \pi / \omega_{m} \ll T_{2 s}^{*} .
$$

При этом в стационарных условиях $t \gg T_{2 s}^{*}$ эксперимента величина $\gamma \mathrm{H}_{2} I_{x s}$ уже не зависит от времени. Здесь индексом $r$ обозначены времена релаксации исследуемых линий, а индексом $s$ - времена релаксации возмущаемых линий.

Если выполняется условие (15), то, в зависимости от того, будут ли $H_{1}$ и $H_{2}$ включены одновременно или попеременно, получаем либо сумму спектров монорезонанса и двойного резонанса ( $H_{1}$ включено постоянно, поле $\mathrm{H}_{2}$ дается импульсами), либо спектр двойного резонанса $\left(H_{1}\right.$ и $\mathrm{H}_{2}$ 
включаются и выключаются одновременно), либо спектр монорезонанса с измененными интенсивностями линий вследствие эффекта Оверхаузера ( $H_{1}$ и $H_{2}$ включаются и выключаются попеременно). Последний спектр свободен от расщеплений, обычно сопровождающих появление ядерного эффекта Оверхаузера. Для получения подобных спектров, кроме (15), необходимо выполнение дополнительных условий

$$
2 \pi / \omega_{m} \gg T_{2 s}, \quad 2 \pi / \omega_{m}<T_{1 r}, \quad 2 \pi / \omega_{m}>T_{2 r}^{*} .
$$

Первое из условий (17) обозначает, что в спектре не должны проявляться другие эффекты двойного резонанса, кроме эффекта Оверхаузера, второе - что изменения интенсивностей линий должны быть измеримы в стационарных условиях, а третье отражает требование того, чтп боковые полосы должны попасть в наблюдаемые линии.

Эти требования $\left(T_{1 r}>T_{2 s}\right)$ далеко не всегда выполнимы. Несмотря на это, данная методика применялась для исследования двойного резонанса $H-\{F\}$ в магнитном поле с несколько уменьшенной однородностью. К сожалению, в результате применения очень низкой (от 0,5 до 1 гц) частоты повторения импульсов отношение сигнал/шум полученных спектров настолько низкое, что применение их для количественных исследований вряд ли возможно.

Если $2 \pi / \omega_{m} \cong T_{2}^{*}, J$, то получаются сложные спектры.

Практически наиболее перспективной при исследовании протон-протонного двойного резонанса является методика разделения времени с высокой частотой повторения импульсов (от 1 до $10 \kappa 2 ц)$ ).

Спектрометр. Экспериментально была исследована применимость метода разделения времени для снятия спектров высокого разрешения водорода и спектров протон-протонного двойного резонанса. Была использована описанная ранее аппаратура [7], измененная в соответствии с требованиями импульсной работы (рис. 1). В данной работе, в отлічие от работы С. Гордона и Дж. Балдешвилера $\left[{ }^{18}\right]$, спиновая стабилиза-

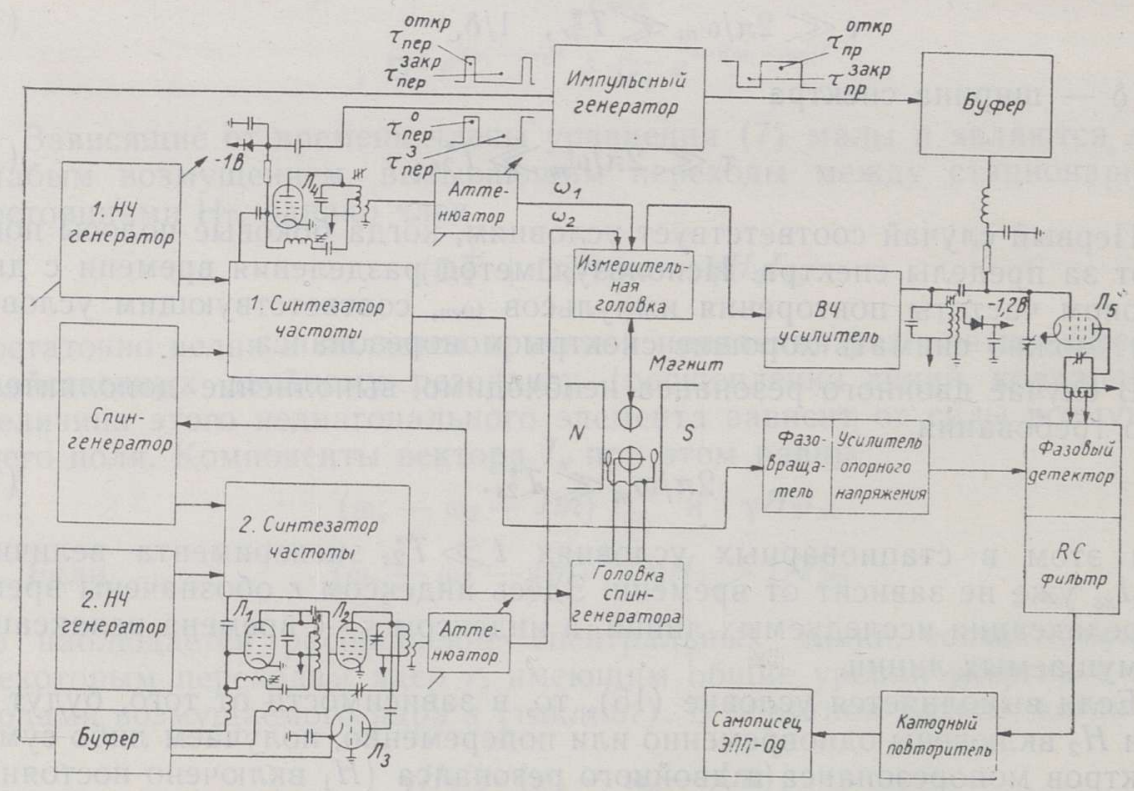

Рис. 1. Блок-схема спектрометра. 
ция осуществлялась с помощью спинового генератора $\left[{ }^{19}\right]$ и, кроме того, обе частоты $\omega_{1}$ и $\omega_{2}$ были привязаны к полю $H_{0}$.

Из выходной частоты спинового генератора синтезируются необходимые частоты $\omega_{1}$ и $\omega_{2}$. Возмущающая частота $\omega_{2}$ преобразуется в последовательность импульсов в выходных каскадах усилителя высокой частоты $J_{1}$ и $J_{2}$, а измерительная частота - в одноламповом усилителе $\pi_{4}$. Чтобы избежать влияния выходного контура с высокой добротностью, последний шунтируется триодом $\mathbb{I}_{3}$, управляемым вместе с усилителем $\boldsymbol{J}_{1}, \boldsymbol{J}_{2}$. Управляющие импульсы получаются из специального импульсного генератора $\left[{ }^{20}\right]$, управляющего обоими блоками синтеза частоты и приемником. При этом возможно одновременное или попеременное включение обоих полей $H_{1}$ и $H_{2}$. В приемнике оказалось достаточным управлять только последним каскадом перед фазовым детектором. Высокочастотное опорное напряжение для фазового детектора подается без импульсной модуляции. Выходное напряжение от фазового детекгора подается через катодные повторители и фильтры на самописец.

Для опытов применялась частота повторения импульсов $\frac{\omega_{m}}{2 \pi}=5 \kappa \imath u$.

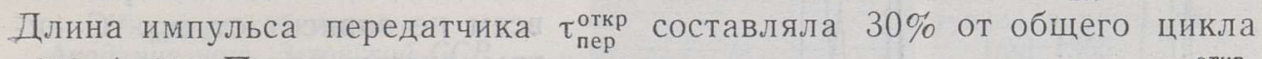
$\tau_{\text {пер }}^{\text {откр }}+\tau_{\text {пер }}^{\text {закр }}$. Приемник закрыт на время, несколько превышающее $\tau_{\text {пер }}^{\text {откр }}$, в течение примерно $50 \%$ от общего цикла.

Так как на образец активно действуют только несущие частоты $\omega_{1}$ и $\omega_{2}$, а не боковые полосы, то в случае применения импульсного метода приходится соответственно увеличивать мощность импульса. В случае прямоугольного импульса с продолжительностью $10 \%$ от общего цикла, уровень высокочастотного напряжения приходится увеличивать в 10 раз.

Результаты опытов. Спектр, снятый методом разделения времени, приведен для сравнения на рис 2. При этом соотношение сигнал/шум

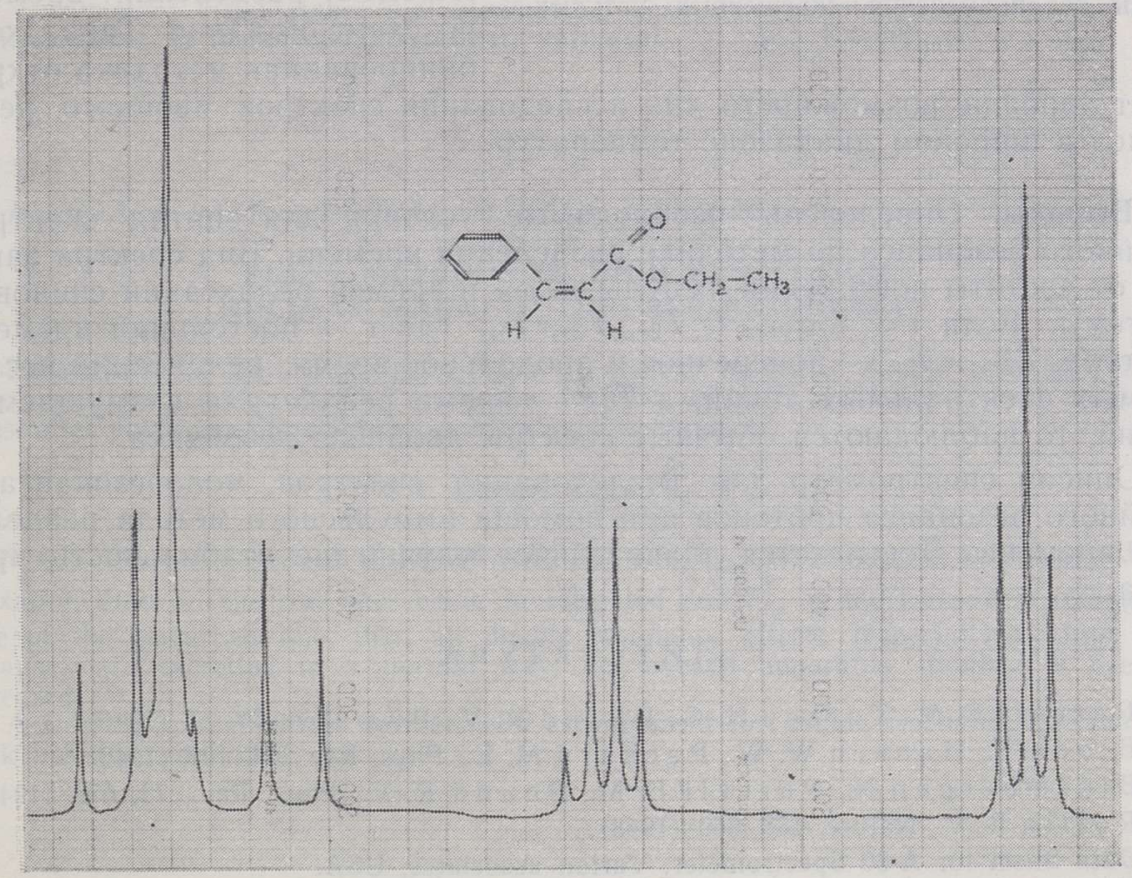

Рис. 2. Спектр этилового эфира коричной кислоты, снятый методом разделения времени (с применением скрещенных катушек, $\omega_{m} / 2 \pi=5 \kappa 2 ц$ ). 


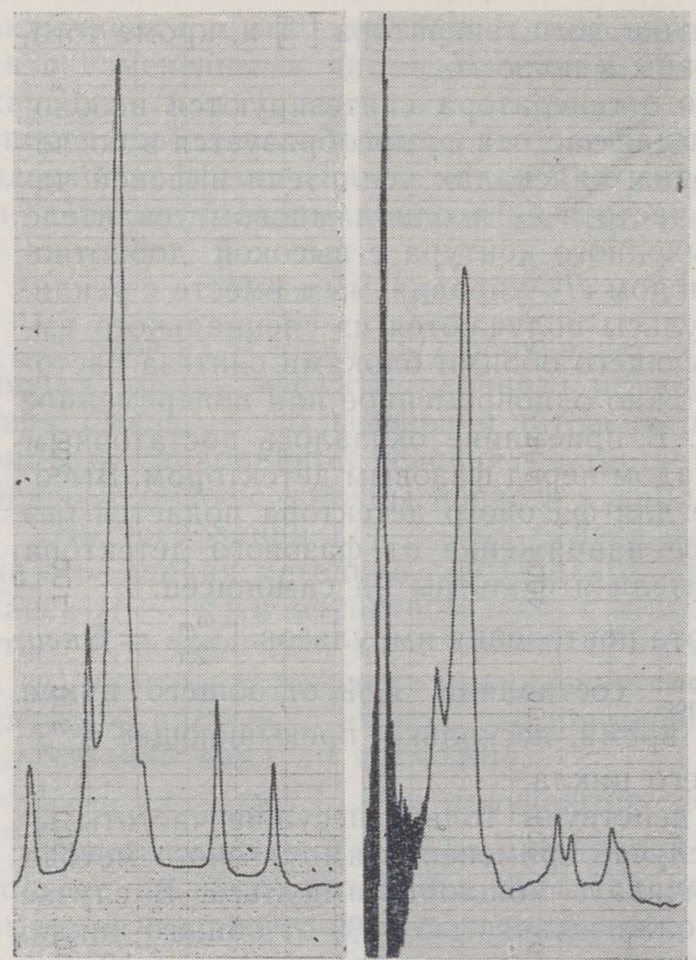

Рис. 3. Спектры кислотного радикала этилового эфира коричной кислоты. Монорезонанс (слева) и двойной резонанс (справа). Спектры сняты без применения скрещенных катушек, $\omega_{m} / 2 \pi=4 \kappa 2 \iota$.

не зависит от относительной длины импульсов $\tau_{\text {пр }}^{\text {отк }}$ н $\tau_{\text {nр }}^{\text {закр }}$, то есть от относительного времени работы приемника. $\mathrm{B}$ идеальном случае такая зави-симость должна отсулствовагь полностью, ибо в стационарных условиях $\left(t \gg T_{2 r}^{*}\right)$ напряжение сигнала и шумовое напряжение не изменяются во время измерительного периода $\tau_{\text {пр }}^{\text {отк }}$. От соотношения $\tau_{\text {пр }}^{\text {отр }} / \tau_{\text {пр }}^{\text {закр }}$, в таком случае, зависит только величина нсобходимого усиления на низкой частоте. Если выполняется усльвие (14), то вид спектра совсем не зависит от $\omega_{m}$. Спектры, снятые при помощи относительно низкой $\omega_{m} / 2 \pi$, равной 400 ги, и при 4 кәи, практически одинаковы.

На рис. 3 приведены спектры монорезонанса и двойноге резонанса (тиклинг) кислотного радикала этилового эфира коричной кислоты, снятые методом разделения времени по схеме Роллина. Такая комбинированная методика открывает хорошие возможности для исследования спектров двойного резонанса в широком диапазоне температур.

Выводы. Определены необходимые условия для снятия спектрог: двойного резонанса по методике разделения времени. Вид спектра зави сит от частоты повторения импульсов $\omega_{m}$ и времен релаксации спиновой системы. Если $\tau \ll 2 \pi / \omega_{m} \ll T_{2 r}^{*}, T_{2 s}^{*}, T_{1 r}$, где $\tau$ - постоянная временін контура, $T_{2 r}^{*}$ и $T_{1 r}$ - поперечное и продольное время релаксации исследуемых спектральных линий, а $T_{2 s}^{*}$ - время релаксации возмущаемой: линии, то наблюдаются обычные спектры двойного резонанса.

Описан спектрометр для исследования спектров монорезонанса и двойного резонанса протонов при помощи импульсного метода разделения времени. Достигается значительное улучшение стабильности прибора.

\section{Л И Т Е Р А Т У Р A}

1. Purcell E. M., Torrey H. C., Pound R. V., Phys. Rev., 69, 37 (1946).

2. Bloch F., Hansen W. W., Packard M. E., Phys. Rev., 69, 127 (1946).

3. Bloembergen N., Purcell E. M., Pound R. V., Phys. Rev., 73, 679 (1948).

4. Roll i n B.. V., Nature, 158, 669 (1948).

5. Data Sheet for A-60 Spectrometer, Varian associates, USA.

6. Data Sheet V-6058. Spin Decoupler for A-60 and A-60A Spectrometers (1965), Variani associates, USA. 
7. Л и п п а а Э., Изв. АН ЭССР. Сер. физ.-матем. и техн. наук, 14, 125 (1965).

8. L a u t e r bur P. C., J. Chem. Phys., 26, 217 (1957).

9. Ли п п а а Э., О ли в с он А., П аст Я., Изв. АН ЭССР. Сер. физ.-матем. и техн. наук, 14, 473 (1965).

10. S a n d s R. H., Ann. of the New York Acad. of Sci., 70 (art. 4.), 780 (1958).

11. D u f f y W., Phys. Rev., 115, 1012 (1959).

12. W a ugh J. S., доклад в Москве.

13. B rownstein S., доклад в Таллине.

14. M e i b o o m S., G i 11 D., Rev. Sci. Instr., 29, 688 (1958).

15. B 1 o ch F., Phys. Rev., 102, 104 (1956).

16. Rabi J. J., R a m s e y N. F., S chw inger J., Rev. Mod. Phys., 26, 167 (1954).

17. Anderson W. A., Freem an R., J. Chem. Phys., 37, 85 (1962).

18. Gordon S. L., B a 1 d e s ch w i e 1 e r J. D., J. Chem. Phys., 43, 76 (1965).

19. Ли п п а а Э., С юг и с А., Изв. АН ЭССР. Сер. физ.-матем. и техн. наук, 14, 129 (1965).

20. Ли ппм а а Э., Паст Я., О лив сон А., С а у вере Т., См. настоящий номер журнала, стр. 58.

21. B a $1 \mathrm{~d}$ e s ch w i e 1 e r J. D., J. Chem. Phys., 34, 718 (1961).

Ннститут кибернетики

Академии наук Эстонской ССР
Поступила в редакцию $1 / X I 1965$

\section{E. LIPPMAA, J. PAST, J. PUSKAR, M. ALLA, A. SUGIS \\ IMPULSSMEETOD SUURE LAHUTUSEGA TUUMA- TOPELTRESONANTSSPEKTRITE UURIMISEKS}

Uurimistöös kirjeldatakse ajajaotusmeetodi kasutamise võimalusi vesiniku tuumadetopeltresonants- ja monoresonantsspektrite uurimisel.

\section{E. LIPPMAA, J. PAST, J. PUSKAR, M. ALLA, A. SÜGIS}

\section{PULSE METHODS IN HIGH RESOLUTION NUCLEAR MAGNETIC DOUBLE RESONANCE}

Possibilities of time sharing methods in nuclear magnetic double resonance have been investigated. The double resonance features of NMR spectrum depend upon the transmitter pulse repetition rate $\omega_{m}$ and relaxation times. If

$$
\tau \ll 2 \pi / \omega_{m} \ll T_{2 r}^{*}, T_{2 s}^{*}, T_{1 r}
$$

where $\tau$ is the time constant of loaded coil, $T_{2 r}^{*}$ and $T_{1 r}$ are transverse and longitudinal relaxation times of spectral lines being investigated and $T_{2 s}^{*}$ is the transverse relaxation: time of the perturbed line, then all double resonance effects (especially tickling and collapse) are exhibited in a normal way, but overall apparatus stability is greatly iricreased.

A suitable spin-stabilized spectrometer arrangement for double resonance time sharing work has been described. 